\title{
25 Research Square \\ Neurobiological Alterations Of The Substantia Nigra In Sudden Infant Death Syndrome
}

\section{Anna Lavezzi ( $\nabla$ anna.lavezzi@unimi.it )}

Universita degli Studi di Milano Facolta di Medicina e Chirurgia https://orcid.org/0000-0002-03775245

\section{Riffat Mehboob}

The University of Lahore

\section{Graziella Alfonsi}

Universita degli Studi di Milano Facolta di Medicina e Chirurgia

\section{Stefano Ferrero}

Universita degli Studi di Milano Facolta di Medicina e Chirurgia

\section{Research}

Keywords: Neuropathology, substantia nigra, brainstem, sudden infant death syndrome, tyrosine hydroxylase, neuromelanin

Posted Date: February 20th, 2020

DOl: https://doi.org/10.21203/rs.2.24091/v1

License: (c) (i) This work is licensed under a Creative Commons Attribution 4.0 International License. Read Full License 


\section{Abstract}

Background - The purpose of this study was to research possible morphological and functional alterations of the substantia nigra in SIDS.

Methods - Brainstems of 46 victims of sudden infant death, aged from 1 to 7 months, were investigated. Twenty-six of these cases were diagnosed as SIDS, due to the lack of any pathological finding, while the remaining 20 cases, in which the cause of death was determined at autopsy, served as controls. Maternal smoking was reported in $10 \%$ of controls and $77 \%$ of SIDS. Histopathological examination of the substantia nigra was done on midbrain 5 - $\mu \mathrm{m}$-thick sections stained with hematoxylin-eosin and KlüverBarrera. Densitometry, immunohistochemistry and histochemistry were applied to highlight the neuronal concentration, the expression of tyrosine hydroxylase and neuromelanin, respectively.

Results - Hypoplasia of the pars compacta of the substantia nigra was observed in SIDS but not in controls. Tyrosine hydroxylase expression in the substantia nigra was significantly higher in controls than in SIDS. Neuromelanin was observed as dark granules only in 4 infants of the control group but never in SIDS. A significant correlation was found between hypoplasia/low neuronal density, low tyrosine hydroxylase expression in the substantia nigra pars compacta and maternal smoking.

Conclusion - The substantia nigra pars compacta, that is the main dopamine center in the brain, is involved in the control of many functions, including the sleep-arousal phase, and hence, its structural and/or functional alterations may explain the pathogenesis of SIDS, that often occurs during sleep. This study also indicates that maternal smoking can may have strongly influenced the $\mathrm{SN}$ altered development.

Trial registration - not applicable for this study

\section{Introduction}

The "substantia nigra" (SN) is a large midbrain structure that lies dorsal to the cerebral peduncles extending from the rostral border of the pons to the subthalamic region. Although the SN looks like a continuous band on both the right and left hand side of transverse histological sections, it is actually composed of two parts with very different connections and functions: the "pars compacta" (SNpc), a dorsal stratum of closely spaced pigmented cells that contain dopamine and neuromelanin (a product of the dopamine synthesis), and the "pars reticulata" (SNpr), a larger ventral region of widely scattered and mainly GABAergic cells. Sometimes a third region, the pars lateralis (SNpl), is mentioned in literature, but it is more usually classified as a component of the $\operatorname{SNpr}(1,2)$.

The SNpr conveys output signals from the basal ganglia to many other brain structures which use GABA (Gamma-AminoButyric Acid) as neurotransmitter (such as thalamus and superior colliculus), while the SNpc serves as an input to the basal ganglia circuit, supplying the striatum with dopamine (3-6). 
Moreover, the SNpc dopaminergic neurons play an important role in controlling many brain functions, especially voluntary movement $(7,8)$. Therefore this region is associated with the motor abnormalites commonly observed in patients with Parkinson's disease (PD), in which the degeneration of dopaminergic cells of the SNpc represents the most consistent pathological finding $(9,10)$.

Several studies have reported that, apart from controlling motor functions, dopamine also regulates the sleep-wake cycle (11-13). Dopamine deficiency in the SNpc can therefore lead to sleep disturbances, such as excessive daytime sleepiness and REM (rapid eye movement) sleep behavior disorder which are common in patients with PD $(14,15)$.

Since sleep arousal disorders have frequently been involved in the pathogenetic mechanism of sudden infant death syndrome (SIDS) (16-18), the aim of this study is to evaluate possible developmental defects of SN in a large group of infants who died suddenly and unexpectedly in the first months of life. In particular, using immunohistochemical and istochemical techniques, we aimed to examine the cytoarchitecture of the $\mathrm{SN}$ in these cases and the expression of thyrosine Idroxylase (TH), which is an essential enzyme for the dopamine synthesis (19), as well as neuromelanin (NM), which is known to be a specific marker of dopaminergic neurons (20).

\section{Methods}

We investigated the expression and the stages of development of both TH and NM in the neurons of the $\mathrm{SN}$ in a cohort of 46 sudden infant death syndrome victims, 26 males and 20 females, aged between 1 and 7 months. The post-mortem examination was performed in accordance with the guidelines provided by the Italian law n.31/2006 "Regulations for Diagnostic Post Mortem Investigation in Victims of sudden Infant Death Syndrome (SIDS) and Unexpected Fetal Death" (21).

Consent - The parents of all of SIDS victims included in the study provided written informed consent to autopsy and related research; study approval was granted by the institutional review board of the Milan University (Lino Rossi Research Center).

After the anatomopathological examination, 26 infant deaths were classified as "SIDS", due to the lack of pathological findings. A precise cause of death was formulated at autopsy for the remaining 20 cases, which as they shared certain sociodemographic characteristics (gender, ethnicity and age) at the time of death with the SIDS victims, were used as "controls". For these remaining 20 cases the diagnoses were: congenital heart disease ( 9 cases), severe bronchopneumonia (4 cases), pulmonary dysplasia (3 cases), myocarditis ( 2 cases), malaria (1 case), and pericarditis ( $\mathrm{n} .1$ case).

Before performing the autopsies, a complete clinical history, including information related to death circumstances, was collected for each victim. Twenty-six newborns, including 24 SIDS and 2 controls, died during sleep. In addition, the mothers were asked to complete a questionnaire concerning their smoking habits. Moreover, the guidelines of the Lino Rossi Research Center foresee the removal of a lock of the victims' hair to test for xenobiotics and in particular cotinine, the main metabolite of nicotine, 
characterized by a long elimination half-life, using gas chromatography/mass spectrometry (GC/MS) (22). Twenty of the 26 SIDS victims' mothers (77\%) admitted to being active smokers or it was detected through a nicotine test. The remaining 6 mothers had never smoked, which was verified through the same analysis. Only two of the 20 mothers in the control group (10\%) had a proven smoking habit. Information on cigarette smoking was also collected from fathers, with a positive match for 8 fathers in the SIDS group and 3 in the control group. A summary of the case profiles is shown in Table 1.

Table 1

Case profiles of the study

\begin{tabular}{|lll|}
\hline & SIDS & Controls \\
\hline Number of cases & 26 & $\begin{array}{l}\text { 20 } \\
\text { Diagnosis: }\end{array}$ \\
$\begin{array}{lll}\text { Severe broncopneumonia (n.4); congenital heart diseases (n.9); } \\
\text { pulmonary dysplasia (n.3); myocarditis (n.2); malaria (n.1) }\end{array}$ \\
\hline Sex, male/female & $15 / 11$ & $11 / 9$ \\
\hline Age range (months) & $1-7$ & $2-7$ \\
\hline Dead during sleep & 24 & 2 \\
\hline $\begin{array}{l}\text { Maternal smoking } \\
\text { (smokers/nonsmokers) }\end{array}$ & $20 / 6$ & $2 / 18$ \\
\hline $\begin{array}{l}\text { Paternal smoking } \\
\text { (smokers/nonsmokers) }\end{array}$ & $8 / 18$ & $3 / 17$ \\
\hline
\end{tabular}

\section{Antomopathological procedures}

The study focused on the examination of the brainstem, where the control centres for vital body functions are located. The procedure, which has already been described in depth in some of our previous articles $(23-25)$, is again summarized below.

Figure 1 shows the methodology used for the brainstem processing. On the right, the sampling of four specimens is illustrated. The first specimen, ponto-mesencephalic, includes the upper third of the pons and the adjacent portion of caudal mesencephalon. The second specimen extends from the upper portion of the medulla oblongata to the adjacent caudal portion of the pons. The third specimen includes the obex. A fourth specimen is taken from the rostral tract of spinal cord.

Transverse serial sections from each specimen were made at intervals of $60 \mu \mathrm{m}$. For each level, $5-\mu \mathrm{m}-$ thick sections were obtained, some of which were stained with hematoxylin-eosin and Klüver-Barrera for the histological examination. The remaining sections were saved and stained for further histochemical and immunohistochemical investigations on neurochemical alterations (26), as deemed necessary. 
The slides were observed under a Nikon Eclipse E800 light microscope (Nikon Corporation, Tokyo, Japan) equipped with an ocular micrometer and images of interest were captured using a 40x objective lens and a Nikon Coolpix 8400 digital camera attached to the microscope, using the same settings and exposure times. More specifically, prior to image capturing, the camera was white balanced and the exposure times were standardised to $0.055 \mathrm{~ms}$.

On the left Fig. 1 shows the representative histological sections obtained from the above described specimens and indicates several of the main nuclei and structures to be examined due to their frequent involvement in SIDS in terms of delayed development (hypoplasia/agenesis).

Essentially, the most important centers are: the locus coeruleus, the Kölliker-Fuse and the median raphé nucleus in the first rostral pontine specimen; the substantia nigra and the red nucleus in the midbrain; on the parafacial/facial complex, the superior olivary complex, the retrotrapezoid and the magnus raphé nucleus in the second sample from the caudal pons, on the hypoglossus, the dorsal motor vagal, the ambiguus, the pre-Bötzinger, the inferior olivary, the obscurus and pallidus raphé, the arcuate nuclei and the solitary tract complex in the third medullary sample. A meticulous neuropathological examination was carried out on the substantia nigra (SN), which was the main focus of this study.

\section{Quantification of neuron density in SN}

For each case a densitometric analysis was performed on Klüver-Barrera stained midbrain sections at the root of the oculomotor nerve on one of the two symmetrical sides using an image analyzer (Image-Pro Plus - Media Cybernetics, Silver Spring, Maryland, USA), after plotting the outline of the two portions of the $\mathrm{SN}$, the densely packed dorsal region (namely the SNpc) and the widespread ventral pars (the SNpr). Images of the stained histological sections were acquired using a colour video camera and displayed in a PC-monitor. Only neurons with clearly defined boundaries were considered. The neuronal density of each of the two SN portions (SNpc and SNpr) was expressed as the number of neurons per $\mathrm{mm}^{2}$. The overall data obtained from both SIDS and Controls were expressed as mean values and standard deviation (mean \pm SD).

\section{Tyrosine hydroxylase $(\mathrm{TH})$ immunohistochemistry of the SNpc neurons}

$\mathrm{TH}$, the rate-limiting enzyme in dopamine biosynthesis, was used as a dopaminergic neuronal marker. More specifically, for the immunohistochemical study of neuronal dopaminergic population within the SNpc, tissue sections obtained from the first cranial brainstem sample at caudal midbrain level were incubated with rabbit anti-TH primary antibody diluted in PBS and reacted overnight at $4{ }^{\circ} \mathrm{C}$. Biotin conjugated secondary antibody incubation (1:200 cat \#S-1000 Vector Laboratories) was performed for $30 \mathrm{~min}$ at room temperature. After several washes in PBS, antibody complex was localized using the ABC system (Vectastain ABC Elite kit cat \#PK6101, Vector Laboratories) followed by 3,3'-diaminobenzidine reaction. The sections were then counterstained with Mayer Hematoxilin for nuclei and coverslipped after dehydration in ascending concentrations of ethanol and cleared in xylene. Negative controls were 
performed by pre-absorbing the primary antibody with a relative antigen excess $\left(100 \mu \mathrm{g} \mathrm{mL}^{-1}\right)$ and incubating the complex with the sections in the specific step, which always resulted negative.

\section{- Quantification of TH immunohistochemical results}

In order to quantify the TH-immunopositive neurons in the $\mathrm{SN}$ a qualitative rating system was applied to the two portions of the SN (SNpc and SNpr). A four-point scale was used to quantify the percent of THimmunopositive neurons in the delineated areas:

$0=$ no $\mathrm{TH}$-immunopositivity in the neurons

$1=$ immunopositivity present in $\leq 20 \%$ of the neurons

$2=$ immunopositivity present in $\otimes 20 \%$ and $\leq 50 \%$ of the neurons

$3=$ immunopositivity present in $\otimes 50 \%$ of the neurons

\section{Neuromelanin staining of the SNpc neurons}

The Schmorl' reaction taken from Lillie's method (27) was used to stain the histological sections adjacent to those employed for other examinations, as a reducing method for determining the presence of NM in the neurons. Firstly the sections were treated with a ferric-ferricyanide solution for 5-10 minutes and then washed under running tap water to ensure that all of the residual ferricyanide had been removed, after which a counterstain in Nuclear Fast Red Stain, Kernechtrot was carried out for 5 minutes. Since melanin is able to reduce ferricyanide to ferrocyanide with the production of Prussian blue in presence of ferric salts, the NM appeared dark blue in the neuron cytoplasms under the microscope, while the nuclei were stained in red.

\section{Statistical methods}

All of the evaluations were scored by two independent blinded pathologists. The results were compared with the $\mathrm{K}$ Index $(\mathrm{KI})$ in order to evaluate the inter-observer reproducibility. The Landis and Koch methodology (28) for the interpretation of the $k$ coefficient was then used, where 0 to 0.2 indicates slight agreement, 0.21 to 0.40 indicates fair agreement, 0.41 to 0.60 indicates moderate agreement, 0.61 to 0.80 indicates strong or substantial agreement, and 0.81 to 1.00 indicates very strong or almost perfect agreement (a value of 1.0 implying perfect agreement). A very satisfactory KI value $(0.88)$ was obtained in this study.

The statistical significance of the direct comparisons between groups of victims was determined by analysis of variance (ANOVA). Statistical calculations were carried out using the SPSS (statistical package for social science) statistical software. Differences were considered statistically significant if the $p$ value was $<0.05$. 


\section{Results}

- Morphological examination of the SN

The histological examination of the $\mathrm{SN}$, the main focus of this study, was performed on transversal serial sections of the caudal midbrain stained with Klüver-Barrera. In the control group, this structure was located adjacent to the superior peduncles, with a bilaterally symmetrical "strip-like" shape. Figure 2 highlights the localization of the SN and its components according to the human brainstem atlas of Olszewski and Baxter (29), i.e. the pars compacta (SNpc), with a densely packed and numerous population of neurons, subdivided into subnucleus $\alpha$, and subnucleus $\beta$, with a more rarefied pattern of neurons, and the pars reticulata (SNpr), a relatively poor cell structure lying ventral to the SNpc, which is much larger and more ovoid in shape than the SNpc. The soma of the neurons od the SN are polygonal or fusiform in shape with a light, frequently eccentric nucleus, evident nucleolus and abundant cytoplasm. The dendrites and axons are well visible and intertwined with each other especially in the SNpc (Fig. 3A). Frequent hypoplasia of the SN mainly affecting the SNpc was observed in 18 of the 26 SIDS cases, with a greatly reduced number of damaged fusiform neurons with rare processes, compared to those of the control group (Fig. 3B). This observation was validated by densitometric evaluation, as reported below. Noteworthy was the frequent presence of mitotic figures (telophases) and binucleate neurons in the SNpc of most SIDS cases (Fig. 4).

- Neuron density

Overall the SIDS cases had a significantly lower neuron density in the SNpc compared to the controls $(p<$ 0.01). The mean values in SNpc were $36.5 \pm 32.7 / \mathrm{mm}^{2}$ for the SIDS group and $92.8 \pm 15.3 / \mathrm{mm}^{2}$ for the control group $(p<0.05)$. The number of neurons per $\mathrm{mm}^{2}$ in SNpr was almost overlapping in the two groups (5.44 \pm 2.43 in SIDS and $5.52 \pm 3.1$ in controls). Table 2 summarizes the densitometric evaluations. 
Table 2

Analysis of the SN neuronal density (cells $/ \mathrm{mm}^{2}$ ) in SIDS and Controls

\begin{tabular}{|lll|}
\hline \multicolumn{1}{|c|}{ SIDS } & CONTROLS \\
\hline SNpc & & \\
\hline Range & $5-100$ & $72-120$ \\
\hline Mean value \pm SD & $36.5 \pm 32^{*}$ & $92.8 \pm 15.3^{*}$ \\
\hline SNpr & & $2-10$ \\
\hline Range & $1-9$ & $5.52 \pm 3.1$ \\
\hline Mean value \pm SD & $5.44 \pm 2.43$ & \\
\hline *Significance related to Controls: $p<0.01$ & & \\
\hline $\begin{array}{l}\text { SNpc = substantia nigra, pars compacta; } \text { SNpr = substantia nigra, pars reticulata; } S D=\text { standard } \\
\text { deviation }\end{array}$ & \\
\hline
\end{tabular}

- TH-immunohistochemistry

The percentage of TH-immunopositive neurons in the control group ranged from 60 to $90 \%$ (score 3 ) in the SNpc (Fig. 5A). Contrastingly, a greater loss of TH immunopositivity in the SNpc neurons was observed in the SIDS victims compared to the controls (Fig. 5B). In fact, the number of positive neurons in SNpc was classified as 0 or 1 (with $\leq 10 \%$ of TH-immunopositive neurons) in 22 of the 26 SIDS cases $(84,6 \%)$. Only a few positive neurons were occasionally found in the SNpr of both the controls and the SIDS cases, which probably migrated from the adjacent SNpc.

- Neuromelanin

In 4 infants over six months of age belonging to the control group, dark cytoplasmatic granules were detected in several neurons of the SNpc, which was indicative of NM content (Fig. 6). Conversely, NM was not found in the SIDS cases, even in the older victims of SIDS.

Correlations of results with maternal smoking

All of the results were associated with maternal prenatal smoking. A significant correlation was observed between SNpc hypoplasia, negativity/low TH immunostaining and maternal smoking $(p<0.01)$. In particular, 16 of the 20 SIDS victims and one of the 2 control subjects with smoker mothers showed THscores $=0$ or 1 in the SN neurons. Conversely, low TH expression was detected only in 4 of the 24 victims with non-smoker mothers (6 SIDS and 18 controls).

Figure 7 summarizes schematically all the results obtained in this study.

\section{Discussion}

To the best of our knowledge this study provides the first direct evidence of SN morphological and functional alterations in SIDS. In particular, since the SN, and precisely the SNpc, is known to be a major 
dopamine producing region of the brain, the loss of dopamine neurons from the SNpc provides us with valuable insight into the pathogenetic mechanism of this syndrome. It is well known that among its various functions, dopamine regulates the sleep-wake cycle (11) and in most cases SIDS occurs during arousal from sleep (16-18).

Dopamine is a neurochemical that, together with adrenalin and noradrenalin, belongs to the catecholamine group. The biosynthesis of these neuromodulators starts with phenylalanine, which is converted into tyrosine by phenylalanine hydroxylase. In turn, tyrosine is hydroxylated by tyrosine hydroxylase (TH) to L-dihydroxyphenylalanine (L-DOPA) which is able to cross the blood-brain barrier and reach the brain parenchyma where it is converted to dopamine (DA) by the enzyme L-aminoaciddecarboxylase especially in the $\mathrm{SN}$ of the midbrain (30).

Much attention has been paid to the involvement of DA in locomotor activity, cognitive functions and sensorimotor integration (7,31-33). The degeneration of dopaminergic neurons within the SNpc has long been known to be a hallmark feature of Parkinson's disease, a neurodegenerative disorder mainly characterized by motor deficits and cognitive impairment (as executive function deficits, attention and learning difficulties, etc.) $(9,10)$. Over the last few years, numerous studies have highlighted the role of DA in many physiological functions such as ventilation and neurological modulation of sleep (34-37). In particular, during the arousal phase from sleep to wakefulness there is an increase in the firing activity of dopaminergic neurons of the SNpc. The pressing need to increase DA synthesis is justified by the fact that physiological arousal requires a radical change in brain activity, as it is characterized by hyperventilation, greater motor activity and increased responsivity to sensory and emotional inputs $(38,39)$. A sudden arousal from sleep is also required as a life-saving reflex in case of hypoxia and/or hypercapnia (due to accidental airway obstruction, severe bronchial disease, prone sleeping position, nicotine absorption, etc.) (40).

Defects in sleep arousal in response to a life-threatening stressor is deemed to be the main cause of SIDS $(17,18)$. Sudden infant death is primarily attributed to respiratory failure caused by physiological increase of the respiratory rate that usually occurs under hypoxic conditions to regularize the plasmatic gas values (41).

The results of our study indicate that the $\mathrm{SN}$, and especially the $\mathrm{SNpc}$, undergoes delayed development in most SIDS victims and a reduced number of neurons sometimes characterized by immature features (hypoplasia). In addition, on using the rate-limiting enzyme TH as a marker for dopaminergic neurons, a significant loss of TH-immunopositive neurons was observed, both in cases with normal or reduced neuronal density, compared to the controls. This observation suggests that in SIDS cases the SN neurons are unable to synthesize and store DA. Our results contrast with those reported by Obonai et al (42). These authors, in a study on the catecholamine neurons in the brainstem of SIDS victims, reported that $\mathrm{TH}$ immunoreactivity was decreased in adrenergic and noradrenergic neurons of the vagal nuclei and area reticularis superficialis ventrolateralis, but not in dopaminergic neurons of the SN. 
In support to our statements, electrophysiological data indicated that the loss of only half of the dopaminergic neurons within the SNpc leads to a significant impairment in sleep-wake parameters (12). We can therefore hypothesize that the deficit of dopaminergic neurons by us demonstrated in the SNpc of SIDS victims may have contributed to fatal outcomes, considering that the majority of the SIDS deaths occurred during sleep. The DA deficit may also have hindered the essential motor response of the newborn to a life-threatening stressor which reduces the amount of oxygen supplied to the brain. There is strong evidence that maternal smoking during pregnancy is a major risk factor for SIDS. In fact, in our SIDS cohort, a very low expression of the TH enzyme, indicative of DA deficit, was detected in $80 \%$ of cases with proven nicotine absorption. Our previous SIDS studies revealed a close correlation between maternal smoking, especially during pregnancy, and defects in the morphological/functional development of various nervous system structures (43-49). This study enhances our knowledge on the devastating effect of cigarette smoking and reinforces our awareness that once nicotine crosses the blood-brain barrier, it can harm and disactivate important brain centers which are crucial to perform the vital activities.

It is important to note the differences in neuronal density observed in the SNpc of newborns compared to the neuronal density of adult subjects. Our results indicate a mean neuronal density in the control infants of $92.8 \pm 15.3 / \mathrm{mm}^{2}$, which is definitely a higher value than that observed in adult humans, as reported in literature. Various studies in fact, carried out on the SN albeit on different topics, reported similar mean values of approximately 20 neurons per $\mathrm{mm}^{2}(50-52)$.

It is well known that the decline in the number of adult SNpc neurons may be due to a progressive loss of neurons, which is an inevitable consequence of normal aging (53-55) and that the numeric atrophy through age affects mainly the noradrenergic locus coeruleus and the dopaminergic substantia nigra $(51,56)$.

Another noteworthy finding of this study concerns neuromelanin (NM), a dark stained pigment which is produced and stored in intracellular granules in specific populations of catecholaminergic neurons of the human brain such as the locus coeruleus and especially the $\operatorname{SNpc}(20,57)$.

Histochemical studies have demonstrated that this dark pigment in the human brain has similar properties to the melanin found in skin, hair and eyes, being insoluble to organic solvents and labelled by silver stains $(58,59)$, which was why it was called "neuromelanin". However, little is known about the biology and the physiological functions of NM. We only know that it is directly biosynthesized from LDOPA, precursor in DA synthesis (20). However, a much debated issue is the involvement of NM in cell iron homeostasis and in the neuroprotective function by sequestration of toxic substances (60-63).

In this study it was observed that the NM in controls is normally unexpressed in the $\mathrm{SN}$ in the first months of life and only begins to appear in the form of cytoplasmatic dark granules in several neurons of the SNpc from 6 months of age onwards. This result is inconsistent with current knowledge that NM pigment in the $\mathrm{SN}$ is not visible under light microscopy in newborns and becomes visible only at 2-3 years of age 
(64). However it is interesting to note that, as reported in one of our previous studies (65), the NM in the locus coeruleus can already be identified in 2-3 month-old infants. As yet we are unable to explain these differences in synthesis times. In this study NM was not detected in the SIDS cases, even in the victims over 6 months of age. It was observed that the NM deficiency was understandably related to the decrease in $\mathrm{TH}$, since both NM and TH are byproducts of tyrosine.

Another interesting observation concerns the frequent presence of mitotic/binucleated neurons only in SIDS cases and not in controls. The pathogenetic mechanism for their formation is still unknown. It is well known that during prenatal nervous system differentiation, neuroblasts undergo mitotic activity and migrate to reach their definitive location, where they differentiate into mature neurons that are characterized by the permanent exit from the cell cycle (66-68). However, according to various authors, if necessary neurons can reactivate, the molecular system which is capable of promoting cell proliferation which was previously blocked (69-72). The activated neurons in the SNpc detected in this study could represent an attempt to divide in order to promote an hyperplastic process as a reaction to noxious stimuli and unfavorable circumstances. The functional significance of these neurons obviously merits further investigation.

\section{Conclusions}

This study highlights for the first time the important involvement of the SNpc in SIDS pathogenesis and, as already reported in our previous studies (43-49), confirms that cigarette smoke absorption may have strongly influenced the results here presented. In fact, all the SIDS cases with decreased neuronal density, negative TH and NM expression and with mitotic neurons in the SNpc were born to smoking mothers.

\section{Abbreviations}

GABA= Gamma-AminoButyric Acid; $\mathrm{DA}=$ dopamine; $\mathrm{GC} / \mathrm{MS}=$ gas chromatography/mass spectrometry; $\mathrm{NM}=$ neuromelanin; $\mathrm{SIDS}=$ sudden infant death syndrome; $\mathrm{SN}=$ substantia nigra; $\mathrm{SNpc}=$ substantia nigra pars compacta; $\mathrm{SNpr}=$ substantia nigra pars reticulata; $\mathrm{TH}=$ thyrosine hydroxylase;

\section{Declarations}

Funding: This research did not receive any specific grant from funding agencies in the public, commercial, or not-for-profit sectors.

Availability of data and material: The datasets used and analysed during the current study are available from the corresponding author on reasonable request.

Code availability: not applicable

Authors' contributions: The contributions of the authors can be described as follows: AML designed the study, developed the methods and wrote the manuscript by the collaborative input and extensive 
discussion with RM and SF. GA designed and developed the applied methodology. All authors read and approved the final manuscript.

Conflicts of interest/Competing interests: The authors declare that they have no competing interests.

Ethics approval and consent to participate: This article has been reviewed and approved by the Institutional Review Board of the "Lino Rossi" Research Center of the Milan University. Parents of all the infants included in the study provided written informed consent to autopsy, related researches and publication of the results, as required by the art. 1 of the Italian law n.31/2006.

Consent for publication: not applicable.

Acknowledgements: The authors thank Dr. Dr. Alessia Ricaldi for the excellent technical collaboration. This study was supported by the Italian Health's Ministry in accordance with the Law 31/2006 "Regulations for Diagnostic Post Mortem Investigation in Victims of Sudden Infant Death Syndrome (SIDS) and Unexpected Fetal Death".

\section{References}

1. Sonne J, Beato MR. Neuroanatomy, Substantia Nigra. In: StatPearls [Internet]. Treasure Island (FL): StatPearls Publishing; 2019 Jan-. Available from: https://www.ncbi.nlm.nih.gov/books/NBK536995/

2. Aubert I, Brana C, Pellevoisin C, Giros B, Caille I, Carles D, Vital C, Bloch B. Molecular anatomy of the development of the human substantia nigra. J Comp Neurol. 1997; 379(1): 72-87.

3. Boyes J, Bolam JP. The subcellular localization of GABA(B) receptor subunits in the rat substantia nigra. Eur J Neurosci. 2003;18:3279-93.

4. Ikeda A. Kotani, N. Koshikawa AR. Cools, Differential role of GABAA and GABAB receptors in two distinct output stations of the rat striatum: studies on the substantia nigra pars reticulata an the globus pallidus. Neuroscience. 2010;167:31-9.

5. Hoffman AF, Gerhardt GA. Differences in pharmacological properties of dopamine release between the substantia nigra and striatum: an in vivo electrochemical study. J Pharmacol Exp Ther.1999;289:455-63.

6. Freeman TB, Spence MS, Boss BD, Spector DH, Strecker RE, Olanow CW, Kordower JH. Development of dopaminergic neurons in the human substantia nigra. Exp Neurol. 1991;113:344-53.

7. Bissonette GB, Roesch MR. Development and function of the midbrain dopamine system: what we know and what we need to. Genes Brain Behav. 2016; 15:62-73.

8. Crocker AD. The regulation of motor control: an evaluation of the role of dopamine receptors in the substantia nigra. Rev Neurosci.1997; 8:55-76.

9. Jankovic J. Parkinson's disease: clinical features and diagnosis. J Neurol Neurosurg 2008;79:36876. 
10. Brooks DJ. Motor disturbance and brain functional imaging in Parkinson's disease.Eur Neurol. 1997; 38[suppl]:26-32.

11. Dzirasa K, Ribeiro S, Costa R, Santos LM, Lin SC, Grosmark A, Sotnikova TD, Gainetdinov RR, Caron MG, Nicolelis MA. Dopaminergic control of sleep-wake states. The Journal of Neurosci. 2006;26:10577-89.

12. Lima MM, Andersen ML, Reksidler AB, Vital MA, Tufik $S$. The role of the substantia nigra pars compacta in regulating sleep patterns in rats. PLoS One. 2007;2:e513.

13. Monti JM, Monti D. The involvement of dopamine in the modulation of sleep and waking. Sleep Med Rev. 2007;11:113-33.

14. Gjerstad MD, Alves G, Maple-Grødem J. Excessive Daytime Sleepiness and REM Sleep Behavior Disorders in Parkinson's Disease: A Narrative Review on Early Intervention With Implications to Neuroprotection. Front Neurol. 2018;9:961.

15. Raggi A, Bella R, Pennisi G, Neri W, Ferri R. Sleep disorders in Parkinson's disease: a narrative review of the literature. Rev Neurosci. 2013;24:279-91.

16. Kahn A, Groswasser J, Franco P, Scaillet S, Sawaguchi T. Sudden infant deaths: stress, arousal and SIDS. Early Hum Dev. 2003;75 [suppl]:S147-66.

17. Kato I, Franco P, Groswasser J, Scaillet S, Kelmanson I, Togari H, Kahn A. Incomplete arousal processes in infants who were victims of sudden death. Am J Respir Crit Care Med. 2003; 168:1298303.

18. Hunt CE. Impaired arousal from sleep: relationship to sudden infant death syndrome. J Perinatol. 1989;9:184-7.

19. Daubner SC, Le T, Wang S. Tyrosine hydroxylase and regulation of dopamine synthesis. Arch Biochem Biophys.2011;508:1-12.

20. Zecca L, Tampellini D, Gerlach M, Piederer P, Fariello RG, Sulzer D. Substantia nigra neuromelanin: structure, synthesis, and molecular behavior. J Clin Pathol: Mol Pathol. 2001; 54:414-8.

21. Constitution of the Italian Republic Law $n^{\circ}$ Regulations for diagnostic post-mortem investigation in victims of sudden infant death syndrome (SIDS) and unexpected fetal death. Official Gazette of the Italian Republic, General Series 2006; 34: 4. Available from: http://users.unimi.it/centrolinorossi/files/gazz_ufficiale.pdf.

22. Kintz P. Gas chromatographic analysis of nicotine and cotinine in hair. J Chromatogr.1992;580:34753.

23. Lavezzi AM, Ferrero S, Paradiso B, Chamitava L, Piscioli F, Pusiol T. Neuropathology of Early Sudden Infant Death Syndrome-Hypoplasia of the Pontine Kolliker-Fuse Nucleus: A Possible Marker of Unexpected Collapse during Skin-to-Skin Care. Am J Perinatol.2019;36:460-71.

24. Lavezzi AM, Ferrero S, Roncati L, Matturri L, Pusiol T. Impaired orexin receptor expression in the Kölliker-Fuse nucleus in sudden infant death syndrome: possible involvement of this nucleus in arousal pathophysiology. Neurol Res.2016;38:706-16. 
25. Lavezzi AM, Poloniato A, Rovelli R, Lorioli L, lasi GA, Pusiol T, Barera G, Ferrero S. Massive Amniotic Fluid Aspiration in a Case of Sudden Neonatal Death With Severe Hypoplasia of the Retrotrapezoid/Parafacial Respiratory Group. Front Pediatr. 2019;7:116.

26. Muhammad N, Sharif M, Amin J, Mehboob R, Gilani SA, Bibi N, Javed H, Ahmed N. Neurochemical Alterations in Sudden Unexplained Perinatal Deaths-A Review. Front Pediatr. 2018;6:6.

27. Bancroft JD. Theory and practice of histological techniques. Brancroft JD and Gamble M (eds) 5th ed. Edinburgh, Churchill Livingstone, 2002;253.

28. Landis RJ, Koch GG. The measurement of observer agreement for categorical data. Biometrics 1977;33:159-74.

29. Olszewski J, Baxter D. Cytoarchitecture of the Human Brainstem. 3rd edition. Büttner-Ennever JA and Horn AKE (eds), Munich, 2014.

30. Fernstrom JD, Fernstrom $\mathrm{MH}$. Tyrosine, phenillanine anc datecholamine synthesis and function in the brain. J Nutr. 2007; 137 (6 suppl 1):1539S-47S.

31. Nieoullon A. Dopamine and the regulation of cognition and attention. Prog Neurobiol. 2002; 67:5383.

32. Nieoullon A, Coquerel A. Dopamine: a key regulator to adapt action, emotion, motivation and cognition. Curr Opin Neurol. 2003;16 (suppl 2):S3-9.

33. Standaert DG, Walsh RR. Pharmacology od dopaminergic neurotransmission. In: Tashjian AH, Armstrong EJ, Golan DE (eds). Principles of Pharmacology: The pathophysiologic basis of drug therapy. Lippincott Williams \& Wilkins, 2011;186-206.

34. Cabezas GA, Israili ZH, Velasco M. The action of dopamine on the airways. Am J Ther. 2003;10:47786.

35. Kolesnikova EE, Serebrovskaya TV. Participation of dopamine in regulation of respiration. Neurophysiology. 1998;30:55-62.

36. Dzirasa K, Ribeiro S, Costa R, Santos LM, Lin SC, Grosmark A, Sotnikova TD, Gainetdinov RR, Caron MG, Nicolelis MA. Dopaminergiccontrol of sleep-wake J Neurosci. 2006;26(41):10577-89.

37. Lima MM, Andersen ML, Reksidler AB, Vital MA, Tufik S. The role of the substantia nigra pars compacta in regulating sleep patterns in rats. PLoS One. 2007;2:e513.

38. Jones BE. Arousal systems. Front Biosci 2003; 8:s438-51. Review

39. Berry RB, Gleeson K. Respiratory arousal from sleep: mechanisms and significance. Sleep. 1997;20:654-75.

40. McGinty D, Szymusiak R. The sleep-wake switch: A neuronal alarm clock. Nat Med. 2000;6: 510-11.

41. Kahn A, Groswasser J, Franco P, Scaillet S, Sawaguchi T. Sudden infant deaths: stress, arousal and SIDS. Early Hum Dev 2003;75 (suppl): S147-66.

42. Obonai T, Yasuhara M, Nakamura T, Takashima S. Catecholamine neurons alteration in the brainstem of Sudden Infant Death Syndrome victims. Pediatrics. 1998;101: 285-8. 
43. LavezziAM, Ottaviani G, Mingrone R, Matturri L. Analysis of the human locus coeruleus in perinatal and infant sudden unexplained deaths. Possible role of the cigarette smoking in the development of this nucleus. Brain Res Dev Brain Res. 2005;154:71-80.

44. Lavezzi AM, Corna MF, Matturri L. Ependymal alterations in sudden intrauterine unexplained death and sudden infant death syndrome: possible primary consequence of prenatal exposure to cigarette smoking. Neural Dev. 2010;5:17.

45. Lavezzi AM, Mecchia D, Matturri L. Neuropathology of the area postrema in sudden intrauterine and infant death syndromes related to tobacco smoke exposure. Auton Neurosci. 2012;166:29-34.

46. Lavezzi AM, Matturri L, Del Corno G, Johanson CE. Vulnerability of fourth ventricle choroid plexus in sudden unexplained fetal and infant death syndromes related to smoking mothers. Int J Dev Neurosci. 2013;31:319-27.

47. Lavezzi AM, Corna MF, Repetti ML, Matturri L. Cerebellar Purkinje cell vulnerability to prenatal nicotine exposure in sudden unexplained perinatal death. Folia Neuropathol. 2013; 51: $290-301$.

48. Lavezzi AM, Ferrero S, Roncati L, Piscioli F, Pusiol T, Matturri L. Nicotinic receptor abnormalities in the cerebellar cortex of sudden unexplained fetal and infant death victims-Possible correlation with maternal smoking. ASN Neuro. 2017;9:1-10.

49. Lavezzi AM. Toxic Effect of Cigarette Smoke on Brainstem Nicotinic Receptor Expression: Primary Cause of Sudden Unexplained Perinatal Death. 2018;6:E63.

50. Abbott RD, Ross GW, Petrovitch H, Masaki KH, Launer LJ, Nelson JS, White LR, Tanner CM. Midlife milk consumption and substantia nigra neuron density at death. Neurology.2016;86:512-9.

51. Robert DA, Nelson JS, Ross GW, Uyehara-Lock JH, Tanner CM, Masaki KH, Launer LJ, White LR, Petrovitchs H. Marinesco Bodies and Substantia Nigra Neuron Density in Parkinson's Disease. Neuropathol Appl Neurobiol. 2017;43:621-30.

52. Ross GW, Petrovitch H, Abbott RD, Nelson J, Markesbery W, Davis D, Hardman J, Launer L, Masaki K, Tanner CM, White LR. Parkinsonian signs and substantia nigra neuron density in decendents elders without PD. Ann Neurol. 2004;56:532-9.

53. Brody H. The aging brain. Acta Neurol Scand. (suppl) 1992;137:40-4.

54. Uylings HBM, West MJ, Coleman PD, De Brabander JH, Flood DG. Neuronal and cellular changes in aging brain. Clark CM and Trojanowski JQ (eds.), Neurodegenerative dementias: Clinical features and pathological mechanisms, McGraw-Hill, Cy, New York, 2000; 61-76.

55. Koukolík F. Structural changes in normal aging of the brain. Cas Lek Cesk. 1989;128:773-6.

56. Manaye KF, Mclntire DD, Mann DM, German DC. Locus coeruleus cell loss in the aging human brain: a non-random process. J Comp Neurol.1995;358:79-87.

57. Fenichel GM, Bazelon M. Studies on neuromelanin. II. Melanin in the brainstems of infants and children. Neurology. 1968;18:817-20.

58. PlumS, Steinbach S, Attems J, Keers S, Riederer P, Gerlach M, May C, Marcus K.

Proteomic characterization of neuromelanin granules isolated from human substantia nigra by laser- 
microdissection. Sci Rep.2016;6:37139.

59. Nicolaus BJ. A critical review of the function of neuromelanin and an attempt to provide a unified theory. Med Hypotheses.2005;65:791-6.

60. Zucca FA, Segura-Aguilar J, Ferrari E, Muñoz P, Paris I, Sulzer D, Sarna T, Casella L, Zecca L. Interactions of iron, dopamine and neuromelaninpathways in brain aging and Parkinson's disease. Prog Neurobio 2017;155:96-119.

61. Aime S, Bergamasco B, Biglino D, Digilio G, Fasano M, Giamello E, Lopiano L. EPR investigations of the iron domain in neuromelanin. Biochim Biophys Acta. 1997;1361:49-58.

62. Bridelli MG, Tampellini D, Zecca L. The structure of neuromelanin and its iron binding site studied by infrared spectroscopy. FEBS Lett. 1999;457:18-22.

63. Bolzoni F, Giraudo S, Lopiano L, Bergamasco B, Fasano M, Crippa PR. Magneticinvestigations of human mesencephalic neuromelanin. Biochim Biophys Acta. 2002;1586:210-8.

64. Fenichel GM, Bazelon M. Studies on neuromelanin. II. Melanin in the brainstems of infants and children. Neurology 1968;18:817-20.

65. Lavezzi AM, Alfonsi G, Matturri L. Pathophysiology of the human locus coeruleus complex in fetal/neonatal sudden unexplained death. Neurol Res. 2013;35:44-53.

66. Marin O, Rubenstein JL. Cell migration in the forebrain. Annu Rev Neurosci. 2003;26:441-83.

67. Ayala R, Shu T, Tsai LH. Trekking across the brain: the journey of neuronal migration. Cell.2007;128, 29-43.

68. Cayre M, Canoll P, James E. Goldman Cell migration in the normal and pathological postnatal mammalian brain. Prog Neurobiol. 2009;88:41-63.

69. McShea A, Wahl AF, Smith MA. Re-entry into the cell cycle: a mechanism for neurodegeneration in Alzheimer disease. Med Hypotheses. 1999;52:525-7.

70. Arendt T. Synaptic plasticity and cell cycle activation in neurons are alternative effector pathways. the 'Dr. Jekylland Mr. Hyde concept' of Alzheimer's disease or the yin and yang of neuroplasticity. Prog Neurobiol. 2003;71:83-248.

71. Zhu X, Siedlak SL, Wang Y, Perry G, Castellani RJ, Cohen ML, Smith MA. Neuronal binucleation in Alzheimer disease hippocampus. Neuropathol Appl Neurobiol. 2008;34:457-65.

72. Zang X. Binucleated neurons in the human brain. Chin Med J (Engl). 1989;102:378-81.

\section{Figures}




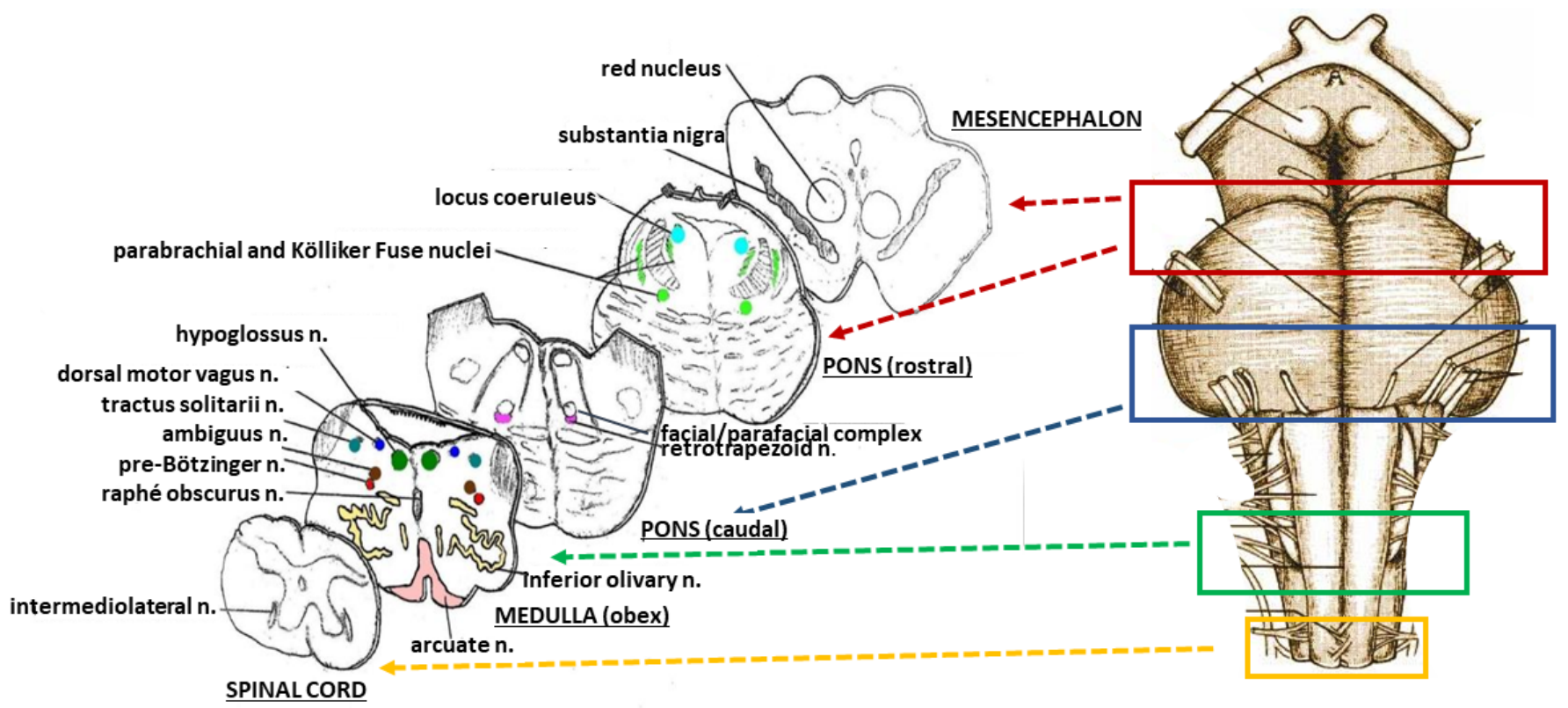

\section{Figure 1}

At the right, sampling of three specimens from the brainstem. The first specimen, ponto-mesencephalic, includes the upper third of the pons and the adjacent portion of mesencephalon. The second extends from the upper portion of the medulla oblongata to the adjacent caudal portion of the pons. The third specimen extends 2-3 mm above and below the obex. A fourth sample is taken from the rostral tract of spinal cord. At the left, the histological sections obtained from the specimens are represented, indicating the main nuclei and structures to be examined. 


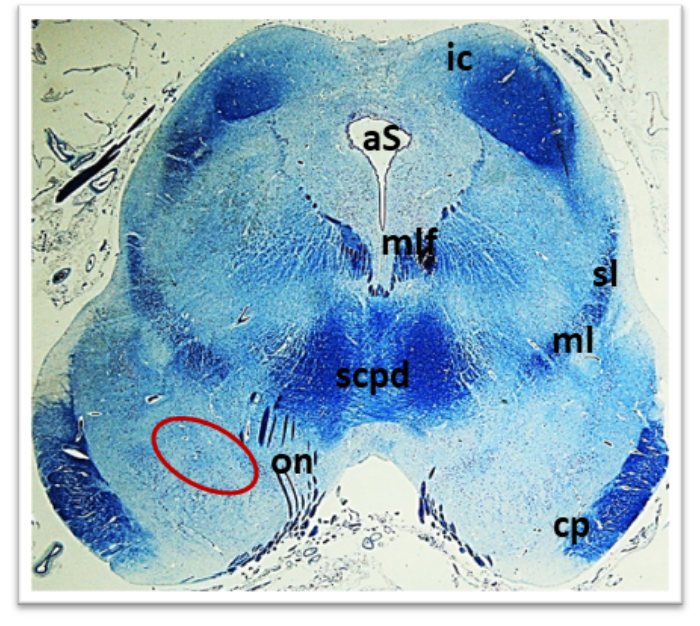

A

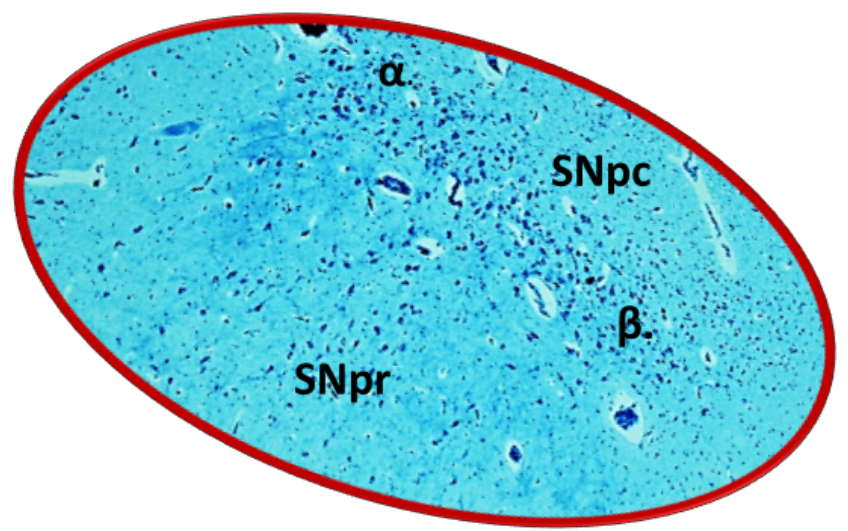

B

\section{Figure 2}

(A) Photomicrographs of a transverse section of midbrain, at the level of inferior colliculus. The circle area, shown at great magnification in (B), indicates the localization of the right part of the bilaterally located substantia nigra. Klüver-Barrera stain. Magnifiction: (A) 0,5x; (B) 10x. aS= aqueduct of Sylvius; $\mathrm{cp}=$ cerebral peuncle; $\mathrm{ml}=$ medial lemniscus; $\mathrm{mlf}=$ medial longitudinal fasciculus; on= oculomotor nervus; ic= inferior colliculus; scpd= superior cerebellar peduncle decussation; sl= spinal lemniscus 


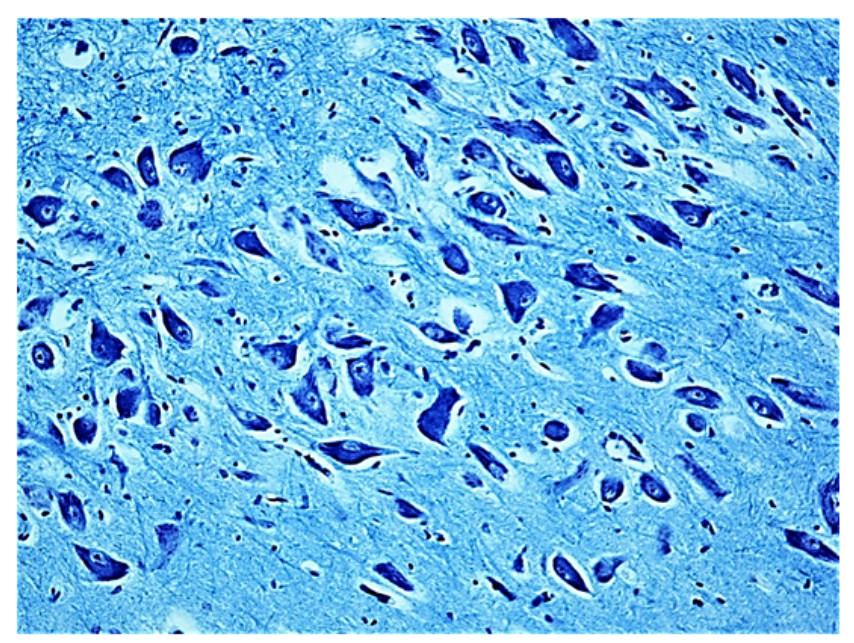

A

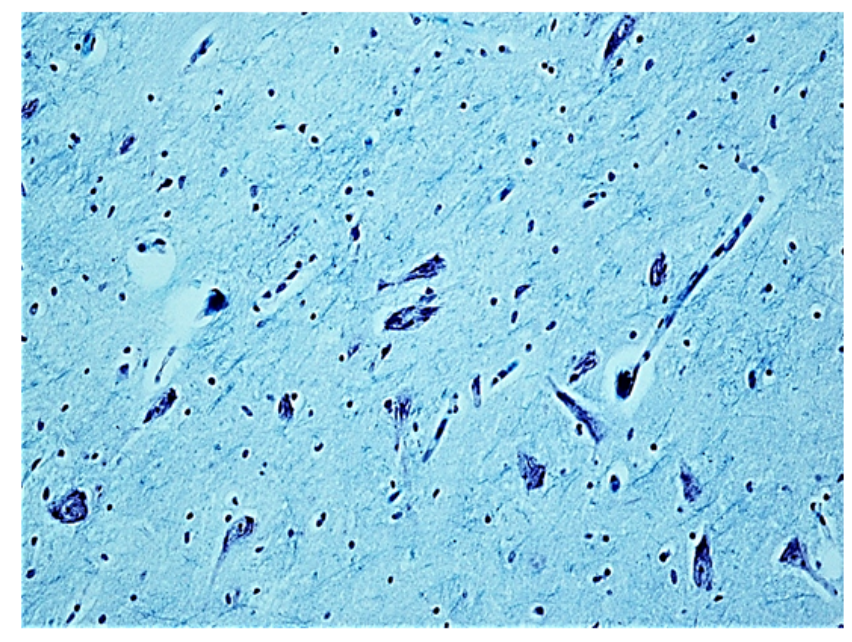

B

\section{Figure 3}

Substantia nigra pars compacta (SNpc). Comparison between the neuron density in a control case (A) and in a case of SIDS (B). Klüver-Barrera stain. Magnifiction: (A) (B) 20x.

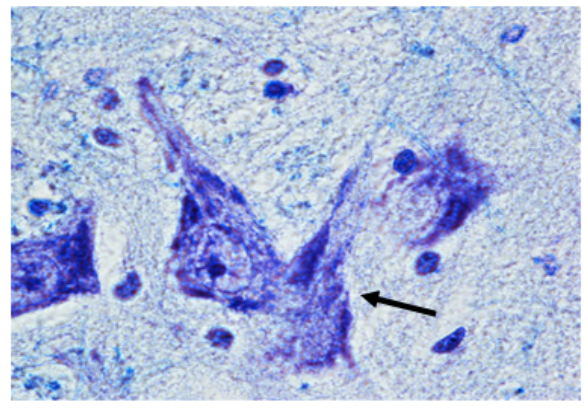

A

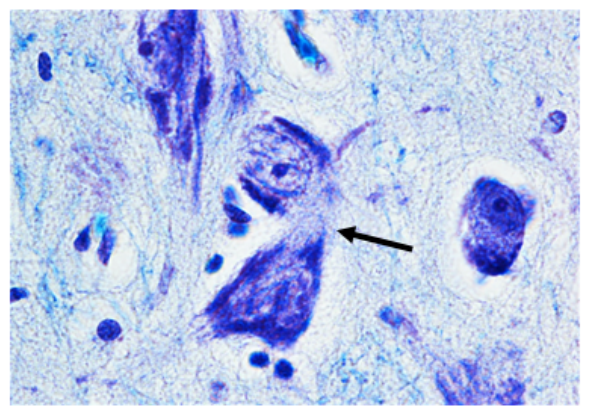

C

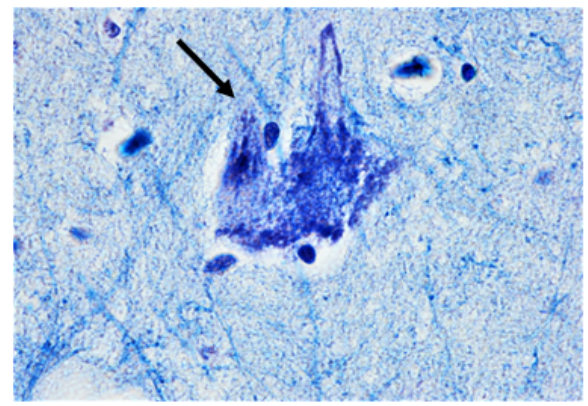

B

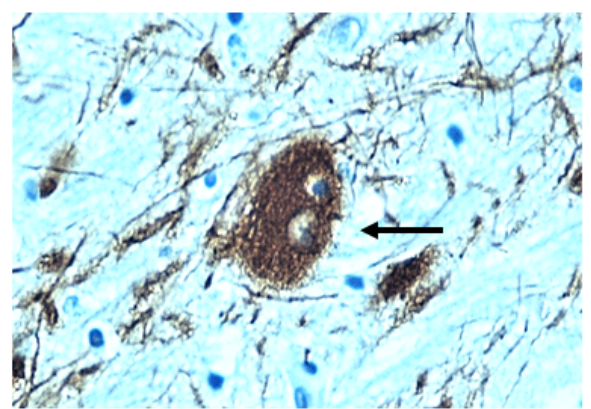

D 


\section{Figure 4}

A panel of photomicrographs showing terminal mitotic figures (highlighted by arrows in $A, B$ and $C$ ) and a binucleate neuron (D) found in the SNpc. A)B)C) Klüver-Barrera stain; C) Thyrosine hydroxylaseimmunohistochemistry. Magnification: 100x.

\section{Figure 5}

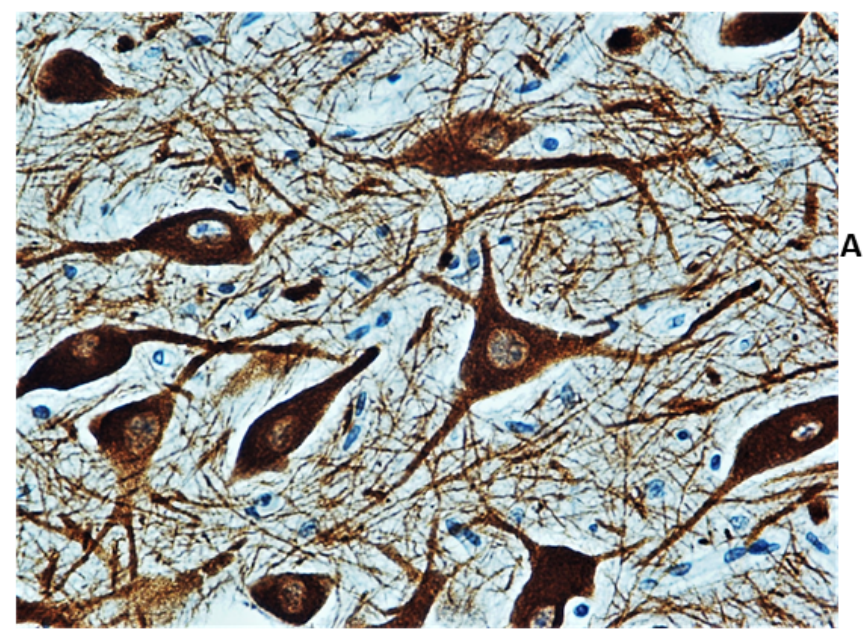

A

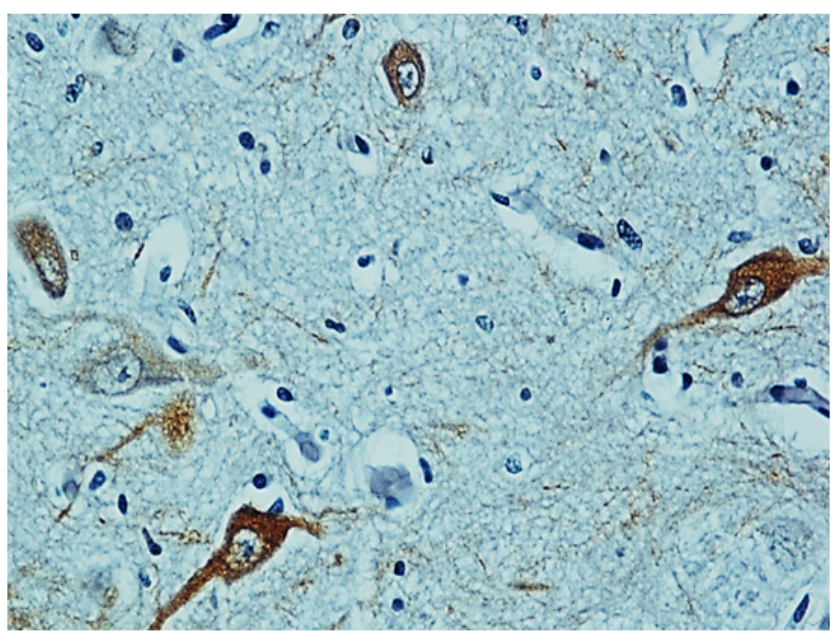

B

\section{Figure 5}

Thyrosine hydroxylase ( $\mathrm{TH})$ - immunohistochemistry. A) intense immunopositivity in the neuronal bodies and processes in the SNpc of a control cases. B) Rare presence of weakly immunostained neurons in the SNpc of a SIDS case. Magnification: 40x. 


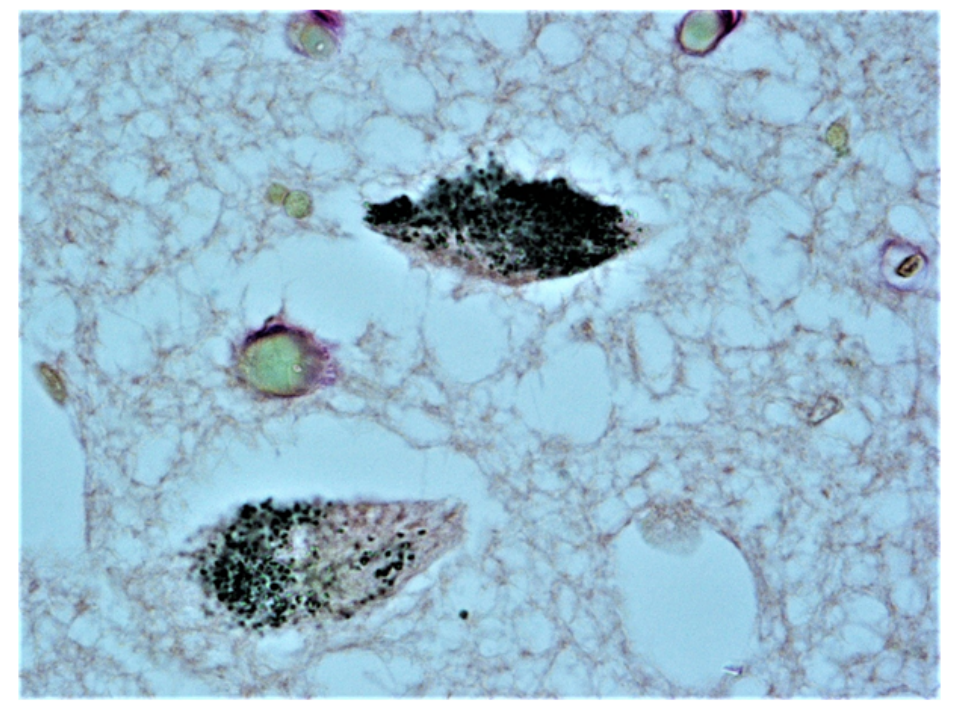

\section{Figure 6}

Neuromelanin (NM) histochemistry. Pigmented neurons in the SNpc of a control infant. Magnification: $40 x$. 

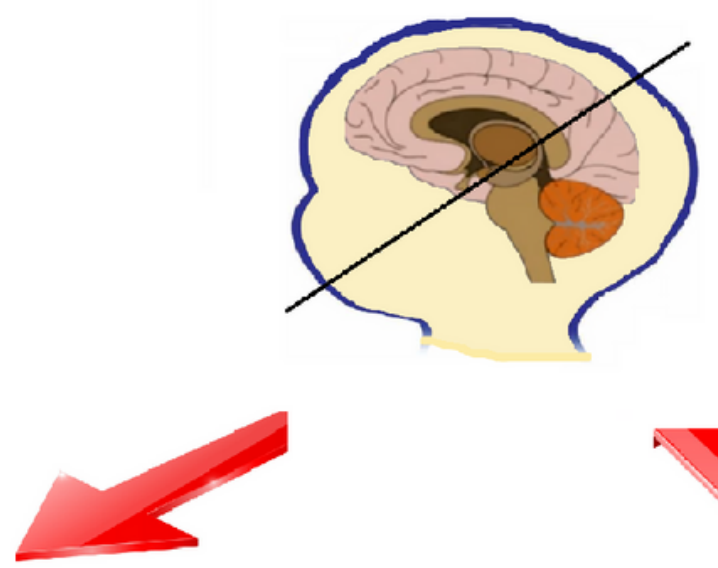

Control

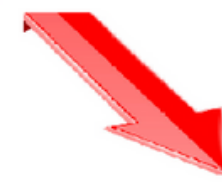

SIDS
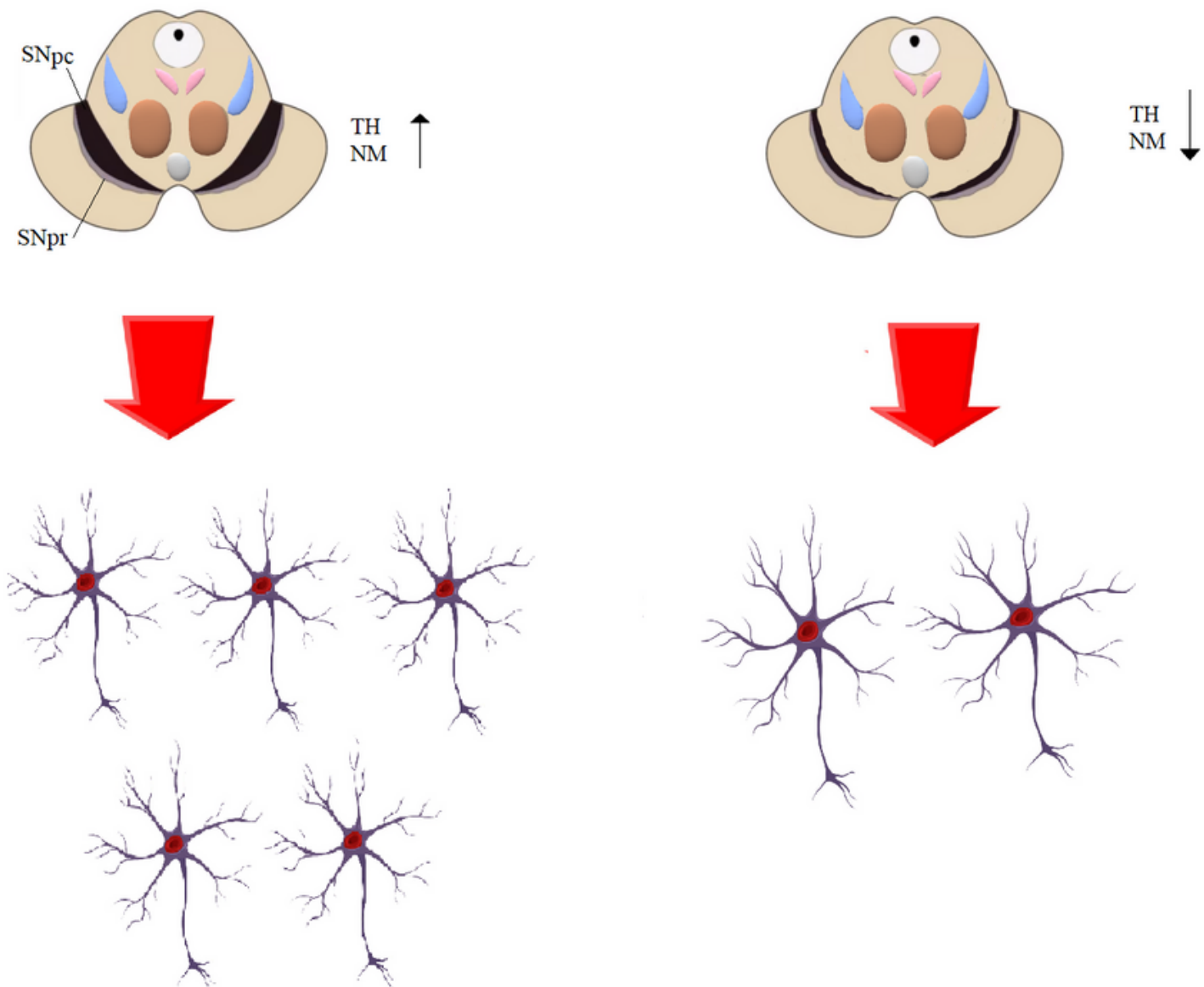

more neuronăl density in SNpc

Non-smoking mothers

less neuronal density in $\mathbf{S N p c}$ Smoking mothers

\section{Figure 7}

Schematic representation of all the results obtained in this study. It's evident that the SN in SIDS cases is characterized by a decrease in the neuron number and in the expressivity of both the TH enzyme and NM with respect to the control cases and furthermore that these results are closely related to the exposure to cigarette smoke. 